\title{
Análisis descriptivo de las creencias y actitudes de alumnos no nativos de español ante los errores y las técnicas de corrección en la interacción oral
}

\author{
Isabel Santos Gargallo \\ Universidad Complutense de Madrid \\ isantos@edu.ucm.es \\ Marcelo Chaparro \\ Global Languages Training-Madrid
}

Resumen: Este artículo explora las creencias o teorías implícitas de los alumnos y su actitud en relación con los errores que tienen lugar en la interacción oral y las técnicas de corrección que los profesores utilizan; profundizar en el conocimiento de las creencias nos ayudará a entender su actitud y a orientar de forma adecuada el proceso de enseñanza-aprendizaje: qué, quién, cuándo y cómo corregir. Nuestro objetivo se concreta en averiguar cuáles son las creencias de los alumnos en relación con los errores en la interacción oral en el aula, y cómo el pensamiento condiciona su actitud y conducta ante las técnicas de corrección del profesor. Desarrollamos un estudio de caso de corte cuantitativo y seudolongitudinal partiendo de un grupo de alumnos con diferentes lenguas maternas y distintas procedencias, $y$ adoptamos la encuesta de respuesta cerrada como técnica para la recogida de datos. El estudio revela que la actitud de los alumnos ante los errores es neutra, pues los consideran algo grave, pero al mismo tiempo necesarios para el aprendizaje; aspecto que se matiza en las conclusiones de este artículo.

Palabras claveः creencias implícitas del alumno, corrección de los errores, interacción oral, español como lengua extranjera. 


\begin{abstract}
This article explores students' beliefs or implicit theories and their attitudes relating mistakes taking place in oral interaction as well as the correction techniques that teachers use. Deepening in the knowledge of assumptions and beliefs will no doubt help us to understand students' attitudes in order to address the learning-teaching process appropriately: what, who, when and how to correct. Our aim is to find out students' range of beliefs regarding mistakes turning out during oral interaction in the L 2 classroom; and how those beliefs determine their attitude and behavior facing teachers' correction techniques. We have carried out a case study, quantitative and pseudo longitudinal type, on students with various native languages and origins. A closed question survey was our data gathering tool. The outcomes reveal students' attitudes towards errors to be of neutral tone for they consider mistakes as serious but, at the same time, necessary for the learning process. Such findings appear to be toned down in the final conclusions.
\end{abstract}

Key words: students' beliefs and implicit theories, error correction, oral interaction, Spanish as a foreign language. 


\section{Introducción}

Este estudio se centra en el pensamiento del alumno. Concretamente se desarrolla en él un estudio de caso - de corte cuantitativo- sobre las creencias y actitudes de hablantes no nativos de diferente procedencia y con distintas lenguas maternas, en relación con los errores y las técnicas de corrección en la interacción oral en el aula de español: quién, qué, cuándo y cómo corregir.

Tras establecer de manera sucinta el estado de la cuestión en relación con los estudios sobre las creencias de los alumnos a propósito de los errores y las técnicas que se aplican para su corrección, explicitaremos los objetivos, la metodología y las características de la muestra de este estudio de caso, para después, llevar a cabo el análisis y discusión de los datos.

\section{Las creencias de los alumnos y los errores en la interacción oral}

\subsection{Las creencias y las actitudes}

La competencia existencial o saber ser del individuo que aprende una lengua está constituida por las actitudes, las motivaciones, las creencias, los valores, los estilos cognitivos y los factores de la personalidad, aspectos que definen junto al saber hacer y el saber aprender, la identidad de quién aprende (MCER, 2002); en este artículo nos centramos en el estudio de las creencias y en cómo estas guían las actitudes.

El estudio de las creencias o teorías implícitas de los alumnos constituye un tema de la adquisición de segundas lenguas, que empieza a ser desarrollado de manera más sistemática por los investigadores a partir de la publicación del test BALLI (Beliefs About Language Learning Inventory ) por Horwitz (1987), si bien los análisis de necesidades y los trabajos sobre estrategias de aprendizaje pueden ser considerados como estudios que abren esta línea de investigación, pues todos ellos se orientan a trazar el perfil del alumno con el fin de guiar el proceso de enseñanza-aprendizaje, ya que «una enseñanza adecuada de la lengua debe ocuparse de los procesos naturales, facilitar y agilizar el aprendizaje, en lugar de impedirlo» (Corder, 1981: 7).

La psicología cognitiva emplea los términos creencias, pensamientos o teorías implícitas, entre otros, para hacer referencia a la representación que tiene un ser humano de la realidad, la cual guía su pensamiento y condiciona su conducta (Abelson, 1979). De hecho, Serra (1997: 155) las denomina representaciones individuales y las define de la siguiente manera: 
... las imágenes o ideas que sobre una cosa se tienen en mente [...] Se trata de apreciaciones subjetivas [...] que acaban por aflorar a través del lenguaje o de las acciones de los individuos. Las representaciones sobre la enseñanza-aprendizaje de lenguas corresponden a la imagen que tienen tanto los enseñantes como los aprendices sobre qué es aprender una lengua, cómo se aprende una lengua y cuándo se puede decir que se sabe una lengua.

Parece claro que las creencias de los profesores se conforman a partir de sus experiencias previas aprendiendo lenguas, su formación y su actuación en el aula; tales aspectos se van incorporando a lo largo de toda la vida y pueden o no modificar su conducta. Además, «muchos profesores tienden a interpretar lo que los alumnos dicen sobre el aprendizaje de la lengua a través del filtro de sus propias creencias» (Picó, Verdés y Vilagrasa, 1990). ¿Y en el caso de los alumnos? ¿Cómo se construyen las creencias del individuo que aprende una lengua segunda o extranjera? ¿Pueden ser consideradas como verdaderas teorías implícitas? Pajares (1992: 310) destaca lo siguiente en relación con algunas de sus características, las cuales pueden ayudarnos a dar respuesta a las cuestiones planteadas:

Las creencias se forman pronto, $y$ tienden a perpetuarse y a mantener su integridad incluso frente a las contradicciones provocadas por la razón, el tiempo, los estudios o la experiencia [... ] Los individuos desarrollan un sistema de creencias que alberga todas las que han ido adquiriendo a través del proceso de transmisión cultural [...] Las estructuras de creencias, tales como las de las creencias educativas, deben entenderse no solo en función de sus interconexiones, sino también de sus conexiones con otras creencias del sistema, tal vez más centrales [...] Resulta insólito que las creencias experimenten cambios en la edad adulta y, cuando esto sucede, la causa más común suele ser un cambio de una autoridad a otra o una alteración terapéutica. [...] Las creencias de los individuos afectan profundamente a su comportamiento.

Si consideramos este elenco de características aplicadas al alumno que aprende una lengua añadida, podemos pensar que sus creencias estarán condicionadas en gran medida por su experiencia previa aprendiendo lenguas - ya sea la materna, una segunda lengua o una lengua extranjera-. Para Picó, Verdés y Vilagrasa (1990) las creencias sobre el aprendizaje de una segunda lengua son, pues, preexistentes y generales; están ahí ya en estado latente y se activan con el aprendizaje concreto de una L2. Sin embargo, cualquiera que haya aprendido distintas lenguas extranjeras en diferentes contextos naturales o educativos podrá afirmar que los procedimientos y estrategias no han sido similares, por lo que ese conjunto de experiencias acumuladas irá conformando y cuestionando sus propias ideas sobre qué significa saber una lengua, cómo aprender una lengua, qué 
papel tienen los errores, qué técnicas prefiere para su corrección en la interacción oral, qué quiere que le corrijan, quién quiere que le corrija, cuándo quiere que le corrijan y cómo quiere que le corrijan, entre otros aspectos. Para poner un ejemplo, si un alumno austriaco ha aprendido su lengua materna —el alemán-, una lengua extranjera como el inglés y una segunda lengua extranjera como el español en un contexto académico de metodología estructural y contrastiva, en el que los errores son considerados como algo negativo, resultará posible que, cuando continúe sus estudios de español en un centro de habla hispana y se adopte una metodología comunicativa y permisiva ante el error, sienta y manifieste rechazo y un cierto apego a su tradición académica: probablemente querrá ser corregido con intensidad y recurrencia.

En este estudio no abordamos el papel del profesor ni sus teorías implícitas en relación con los errores y su corrección, pero no cabe duda de que se trata de un condicionante ineludible de la actitud y creencias de los alumnos (Mekassimove, 2009). El estudio de Díaz Martínez (1998) aborda, desde la perspectiva cualitativa y utilizando el diario introspectivo como instrumento para la recogida de datos, la relación entre los factores afectivos y la actitud ante los errores; $y$ pone de manifiesto que la falta de afectividad y empatía por parte del profesor ante la corrección en la producción oral - especialmente en la pronunciaciónproduce en el aprendiente falta de receptividad, desmotivación e, incluso, rebeldía. Insiste y destaca que lo afectivo puede ser incluso más determinante para el aprendizaje que los propios contenidos. Sin embargo, los resultados no permiten la generalización por tratarse de una sola informante y esta coincidir con la investigadora. En esta misma línea, Fernández de Amo (2008) desarrolla el concepto de error afectivo, para referirse a aquellos errores que no responden a la falta de conocimiento o competencia sino a timidez, inhibición o miedo a exponerse (estos podrían relacionarse con la categoría faltas de Corder, 1967); también Roncel Vega (2008) se interesa en su estudio por la misma correlación analizando el autoconcepto, la motivación y la ansiedad en estudiantes eslavos de español.

Las representaciones o creencias son, a menudo, complejas y difíciles de delimitar, por lo cual permean nuestras maneras de reaccionar y actuar en situaciones de la vida cotidiana de manera más o menos consciente o inconsciente (Groult, 2005). Así, el sistema de creencias de un estudiante guiará su conducta y su actitud ante los errores, tal es el tema de nuestro estudio. En términos generales, puede afirmarse que la actitud es uno de los factores personales que influyen en el aprendizaje de una lengua. Martínez Abellán (1999: 22), manifiesta que la actitud no es un elemento unitario. Se trata de un concepto multiforme que incluye una serie de actividades más o menos específicas. Así pues, podemos diferenciar dos grandes tipos de actitudes que inciden en la adquisición de segundas lenguas: 
a) Actitudes educacionales: sobre el proceso de aprendizaje de lenguas, o más específicas, sobre el aprendizaje de una lengua determinada, sobre el profesor y su estilo, el curso concreto, etc.

b) Actitudes sociales: que tiene que ver con las implicaciones culturales de su adquisición: actitudes sobre las lenguas y sus hablantes tanto desde un punto de vista genérico como específico.

El autor considera que las primeras tienen una incidencia más directa sobre el aprendizaje de las segunda lengua; se trata de una disposición psicológica - mental, intelectual, experiencial, social y afectiva - hacia el proceso de aprendizaje (DTCELE, 2008). Una actitud positiva ante el aprendizaje de lenguas está relacionada con la apertura hacia nuevas experiencias, otras personas, pueblos, sociedades, culturas; con una voluntad de relativizar la propia perspectiva cultural; y con la voluntad y capacidad de distanciarse de las actitudes convencionales en cuanto a la diferencia cultural (MCER, 2002: 103); aspectos, todos ellos, fundamentales, en la relación del que aprende una lengua con los errores y con las técnicas para su corrección adoptadas por los profesores. En este sentido, Horwitz (1987) considera de vital importancia conocer las creencias de los estudiantes, sin lo que un profesor y un programa de enseñanza pueden llegar a frustrar las expectativas de los aprendientes y, por consiguiente, impedir la potenciación de todas aquellas habilidades que no se adquieren en el aula y que forman parte del mundo de las estrategias y del aprendizaje autónomo; en ello insiste también el estudio de Mekassimove (2009), centrado en el contexto de la Educación Secundaria gabonesa.

\subsection{La corrección de los errores en la interacción oral}

El tratamiento de los errores en la interacción oral -intercambio alternante entre dos o más interlocutores que conlleva negociación de significados y respeta el principio pragmalingüístico de cooperación - ha sido objeto de menor atención que la correspondiente en la expresión escrita. Las interacciones orales que se desarrollan en el aula de lenguas extranjeras tienen lugar en forma de preguntas, reformulaciones, comprobaciones, afirmaciones, solicitud de información, repeticiones, opiniones, diálogos, debates, descripciones, narraciones, presentaciones argumentativas y expositivas, $y$ un largo etcétera; $y$ en una cantidad y manera que depende, entre otros aspectos, del tiempo disponible, del número de alumnos, de las características del aula, pero sobre todo, de aspectos de la personalidad de los interlocutores, del currículo y de la metodología adoptada en la enseñanza. En este contexto didáctico, entendemos la corrección de los errores como la aplicación del algún procedimiento didáctico para reparar, mejorar, hacer más efectivo, 
más adecuado, más próximo a la norma cualquier aspecto de la producción del alumno en una situación de interacción en contexto de instrucción formal.

Los errores, desde la perspectiva positiva que adoptamos, son un fenómeno inevitable y necesario, constituyen parte de la interlengua del hablante no nativo y tienen lugar tanto en niveles de iniciación al aprendizaje de la lengua como en otros de dominio más avanzado, en los cuales, suele aparecer el fenómeno de la fosilización (Corder, 1967, 1981) al que nos referiremos más adelante. Si su necesidad, en tanto que elementos característicos, activos y creativos de la interlengua (Selinker, 1972), y su carácter inevitable son indiscutibles, ¿̨resulta necesaria la corrección de los errores? ¿Las técnicas de corrección de errores, adoptadas por los profesores, resultan satisfactorias en modo y cantidad para los alumnos? Acabamos de plantear dos preguntas de aparente sencillez, pero entrañan gran complejidad, y su respuesta debería depender de las necesidades de los alumnos como protagonistas del proceso - aunque no siempre sea así-, de su propósito y motivación en el aprendizaje de la lengua, de los rasgos de su personalidad, de su estilo cognitivo, de sus estrategias de aprendizaje, en fin, del alumno. En términos generales, creemos que la corrección gramatical ha de ser característica de la interlengua, en igual medida que la adecuación, si el que aprende una lengua extranjera se propone utilizarla con fines profesionales que así lo requieran, tales como la enseñanza, la traducción, la interpretación, el turismo, la diplomacia, etc. o con fines académicos; pero, si por el contrario, su motivación está relacionada con establecer relaciones informales con hispanohablantes o viajar por países del ámbito hispano entre otras, primará la comunicación y deberá ser la actitud del aprendiente la que determine quién, qué, cuándo y cómo quiere ser corregido.

De todas las características de la interlengua, una de las más relevantes es el mecanismo de la fosilización, un fenómeno por el que el hablante no nativo tiende a conservar en su IL formas, reglas y subsistemas erróneos, de manera recurrente y en estadios del aprendizaje en que estos esquemas deberían estar superados. Las causas más frecuentes de la fosilización suelen ser la interferencia lingüística entre la L1 y la lengua meta, la interferencia con otras lenguas segundas o extranjeras en las que presenta un alto nivel de competencia comunicativa y las propias estrategias de aprendizaje que, a veces, llevan a realizar generalizaciones erróneas sobre el funcionamiento de la lengua meta. Este fenómeno es más frecuente en hablantes no nativos cuyo objetivo es una competencia comunicativa funcional que les permita manejarse en las situaciones de la vida cotidiana, y en estudiantes que acceden al aprendizaje de una L2/LE en la edad adulta y sin experiencia previa en el aprendizaje de idiomas. 
Desde un punto de vista didáctico, resulta útil la distinción entre errores transitorios, fosilizables y fosilizados a la que alude Fernández López (2000) y que podemos resumir como sigue:

a) Errores transitorios o de desarrollo: son los propios de los diferentes estadios de la interlengua, los cuales tienden a ser superados en etapas posteriores; son el resultado de la formación de hipótesis sobre el funcionamiento de la lengua. A medida que se avanza en el proceso de aprendizaje, se suceden distintas formaciones y contrastes en un bagaje lingüístico cada vez más amplio.

b) Errores fosilizables: son aquellos que ofrecen una especial resistencia, ya sea por la complejidad de la estructura, por el contraste con la lengua materna o por la no interiorización de la regla; resultan especialmente fosilizables aquellos aspectos que presentan un fuerte contraste entre la lengua materna y la lengua meta

c) Errores fosilizados: son aquellos errores que se repiten de forma recurrente, se han fijado en la interlengua y normalmente se corresponden con estadios anteriores del aprendizaje.

En relación con la corrección de estas tres categorías de errores, se puede decir que los errores transitorios, ya que se encuentran en un estadio de desarrollo de la interlengua tal y como su propia denominación refiere, no deberían ser objeto de corrección; más bien deberíamos centrarnos en los fosilizables, que ofrecen una mayor resistencia, debida normalmente a la interferencia con la lengua materna o a su especial dificultad. Así, las técnicas para la corrección de errores pueden centrarse en la forma o en el significado (Fernández López, 2000); sin embargo, esta distinción no suele ser útil para el alumno, para quien un error es un error y los errores son principalmente de tipo lingüístico, creencia que para muchos profesores resulta errónea.

No obstante, no solo las técnicas específicas para la corrección inciden en la actitud de los alumnos ante los errores, Arumi Ribas (2009) en un estudio, en el que utiliza la encuesta semiabierta y la entrevista, y aborda cuatro aulas universitarias de lengua extranjera —inglés, francés, alemán y español一, constata que algunos de los procedimientos de autorregulación —en concreto, la hoja de reflexión, la parrilla de autoevaluación, la tutoría, y la retroalimentación, no así el portafolio- que se emplean en el proceso de enseñanza-aprendizaje en el marco del Espacio Europeo de Educación Superior (EEES), sirven para que el alumno tome conciencia de sus carencias y de sus errores y para adoptar, en definitiva, una actitud positiva ante el proceso. 
En nuestro contexto, el estudio de Barbero Carcedo (2012) puede ser considerado la propuesta más completa sobre las técnicas de corrección de errores en la expresión o interacción oral en el ámbito de la enseñanza del español como lengua extranjera, una de cuyas aportaciones más relevantes la constituye su taxonomía que, partiendo de la bibliografía existente (Long, 1977; Chaudron, 1988), las sistematiza y agrupa en dos variables principales - a) interrumpir al alumno o no y b) dar la solución o no- y da lugar a cuatro grupos de técnicas de corrección diferenciados (ibíd.: 18-20):

\section{Tabla 1: Técnicas de corrección en la interacción oral}

\begin{tabular}{|c|c|}
\hline $\begin{array}{l}\text { Recurro a la comunicación no verbal: un } \\
\text { gesto facial, un movimiento de la mano/ } \\
\text { cabeza, un ruido, un carraspeo, etc. } \\
\text { Llamo la atención sobre el error utilizan- } \\
\text { do expresiones como cuidado, atención. } \\
\text { Llamo la atención sobre el error mediante } \\
\text { interjecciones: ¿eh? } \\
\text { + Pido repetición al alumno. } \\
\text { + Pido aclaración por falta de comprensión } \\
\text { sobre lo que el alumno ha querido expre- } \\
\text { sar. } \\
\text { Repito la producción del alumno, pro- } \\
\text { nunciando interrogativamente la parte } \\
\text { errónea o marcando esta de algún otro } \\
\text { modo. } \\
\text { Repito el enunciado del alumno inte- } \\
\text { rrumpiéndolo en el punto en el que se } \\
\text { encuentra el error para que el alumno lo } \\
\text { complete correctamente. } \\
\text { Repito la pregunta que dio lugar a una } \\
\text { respuesta errónea. } \\
\text { Repito la pregunta que dio lugar a una } \\
\text { respuesta errónea, alterándola sintáctica- } \\
\text { mente. } \\
\text { Llamo la atención sobre el error explícita- } \\
\text { mente: «Rompido» no está bien. }\end{array}$ & $\begin{array}{l}\text { 2. TÉCNICAS EN LAS QUE SE } \\
\text { INTERRUMPE Y SE DA LA SOLUCIÓN } \\
\text { En primer lugar, llamo la atención sobre el } \\
\text { error con alguna de las técnicas anteriores: } \\
\text { + Recurro a la comunicación no verbal: un } \\
\text { gesto facial, un movimiento de la mano/ } \\
\text { cabeza, un ruido, un carraspeo, etc. } \\
\text { Llamo la atención sobre el error utilizan- } \\
\text { do expresiones como cuidado, atención. } \\
\text { Llamo la atención sobre el error mediante } \\
\text { interjecciones: ceh? } \\
\text { Repito la producción del alumno, pro- } \\
\text { nunciando interrogativamente la parte } \\
\text { errónea o marcando esta de algún otro } \\
\text { modo. } \\
\text { Llamo la atención sobre el error explícita- } \\
\text { mente: «Rompido» no está bien. } \\
\text { En segundo lugar, ofrezco la forma correcta de } \\
\text { alguna de estas maneras: } \\
\text { Doy la versión correcta. } \\
\text { Doy la versión correcta y hago que la } \\
\text { persona que cometió el error repita la } \\
\text { corrección. } \\
\text { Doy la versión correcta y hago que toda la } \\
\text { clase repita la corrección. } \\
\text { Doy una explicación metalingüística so- } \\
\text { bre el error. }\end{array}$ \\
\hline
\end{tabular}




\begin{tabular}{|c|c|c|}
\hline $\begin{array}{c}\text { 3. TÉCNICAS EN LAS QUE NO } \\
\text { SE INTERRUMPE Y NO SE DA LA } \\
\text { SOLUCIÓN }\end{array}$ & $\begin{array}{c}\text { 4. TÉCNICAS EN LAS QUE NO SE } \\
\text { INTERRUMPE Y SE DA LA SOLUCIÓN }\end{array}$ \\
$\begin{array}{c}\text { Al final de la clase o de la actividad escri- } \\
\text { bo en la pizarra algunos enunciados erró- } \\
\text { neos que he escuchado y apuntado, y pido } \\
\text { a los alumnos que encuentren los errores } \\
\text { y los corrijan. }\end{array}$ & $\begin{array}{l}\text { No llamo la atención sobre el error pre- } \\
\text { viamente sino que reformulo la idea que } \\
\text { quiere comunicar el alumno con la forma } \\
\text { correcta. Mi intervención se integra en la } \\
\text { conversación. }\end{array}$ \\
$\begin{array}{l}\text { Espero a que el alumno acabe de hablar } \\
\text { para llamar la atención sobre el error y } \\
\text { darle la corrección. } \\
\text { Al final de la clase o de la actividad escri- } \\
\text { bo en la pizarra algunos enunciados que } \\
\text { he escuchado y apuntado, y los corrijo. }\end{array}$ \\
\hline
\end{tabular}

A estas técnicas podrían añadirse otras, como la grabación audiovisual, ante la que los alumnos manifiestan distintos grados de aceptación. Nuestro estudio empírico integra algunas de estas propuestas, pero no todas ya que, como veremos a continuación, no solo se centra en el cómo y cuándo corregir sino también en otros aspectos, y hubiera resultado exhaustivo incluirlas todas.

\section{Objetivos}

Esta investigación se propone como objetivo general averiguar cuáles son las creencias de los alumnos en su relación con los errores en la producción oral, y cómo el pensamiento condiciona su actitud y conducta ante las técnicas de corrección; dicho objetivo se concreta en los siguientes específicos:

a) Actitud positiva o negativa ante los errores.

b) Actitud positiva o negativa ante la corrección de los errores en el aula.

c) Preferencias de los alumnos en relación con quién corrige.

d) Preferencias de los alumnos en relación con qué corregir.

e) Preferencias de los alumnos en relación con cuándo corregir.

f) Preferencias de los alumnos en relación con cómo corregir.

\section{Metodología de la investigación}

Los estudios sobre las teorías implícitas y las creencias del profesor y/o del alumno pueden ser abordados desde cualquiera de los dos paradigmas científicos - cuantitativo y cualitativo - o combinar ambos, ya que, de acuerdo con Nunan (1992), creemos que en lingüística aplicada y adquisición de segundas lenguas (ASL), la adopción de métodos y técnicas adscritas a ambos o a cualquiera de 
los dos puede resultar provechosa y proporcionar una visión más completa sobre cuestiones en las que el informante y proveedor de los datos es un sujeto complejo y los fenómenos que se investigan, poliédricos: estos dos paradigmas no resultan excluyentes sino complementarios, no existe cisma entre ellos, tal y como afirman Larsen Freeman y Long (1994:32), consideramos que «tenemos mucho que ganar aproximándonos al estudio de la ASL combinando las ventajas de los paradigmas cuantitativo y cualitativo. En vez de considerarlos como paradigmas opuestos, entendemos que son complementarios, y con ello implicamos que no hay que optar necesariamente por uno u otro».

Algunas de las investigaciones sobre el pensamiento del profesor y/o del alumno han sido abordadas desde la perspectiva cualitativa, priorizándose en ellas los aspectos descriptivos y exploratorios; no obstante, en este estudio, hemos decidido acogernos al paradigma cuantitativo — positivista, objetivo, medible, verificable y orientado hacia los resultados - con el fin de abordar una muestra más amplia y aumentar, así, la capacidad de generalización y la posible contrastación del estudio.

En el marco del paradigma cuantitativo, adoptamos el estudio de caso como método que «describe el comportamiento y la actuación lingüística de un número de hablantes a fin de generalizar las conclusiones al total de la población» (Yin, 1984: 14). Un método empírico, selectivo y descriptivo que estudia un fenómeno - en el nuestro, las creencias de los alumnos-, dentro de un contexto real de la vida cotidiana. Además, se trata de un estudio de caso de carácter seudolongitudinal, ya que la muestra está constituida por alumnos de tres cursos consecutivos, pero los datos han sido tomados de forma simultánea durante el mismo año académico.

En cuanto a la técnica adoptada para la compilación de los datos, hemos elegido el cuestionario de respuesta cerrada, que tal y como apuntan Richards y Lockhart (1998: 19) resulta «útil para recopilar información sobre las dimensiones afectivas de la enseñanza y el aprendizaje, tales como puntos de vista, actitudes, motivación y preferencias». El cuestionario consta de 36 variables: las 10 primeras establecen el perfil del informante, y las 26 restantes abordan las creencias y actitudes de los alumnos ante los errores y las técnicas empleadas en su corrección en la interacción oral.

\section{Muestra}

La muestra — no aleatoria sino casual — está constituida por 143 alumnos de los niveles B1.1., B1.2. y B2 (intermedio 1, curso cuarto y curso quinto) de la Escuela 
Oficial de Idiomas de Madrid "Jesús Maestro» ${ }^{1}$. La compilación de los datos se llevó a cabo en los dos horarios ofertados por el centro: mañana y tarde; los informantes dispusieron de quince minutos para cumplimentar el cuestionario; y el absentismo fue del $5 \%$ del total de los matriculados en la modalidad presencial ${ }^{2}$.

A continuación destacamos los aspectos más relevantes del análisis ${ }^{3}$ en relación con el perfil de los informantes que constituyen la muestra:

\section{Tabla 2: Perfil de los informantes}

\begin{tabular}{|c|c|}
\hline $\begin{array}{l}\text { Nivel de referencia } \\
\text { en español }\end{array}$ & $\begin{array}{l}\text { B1.1: 66,4\%. } \\
\text { B1.2: } 21 \% \\
\text { B2: } 12,6 \%\end{array}$ \\
\hline Edad & $\begin{array}{l}\text { De } 15 \text { a } 19 \text { años: } 7 \% . \\
\text { De } 20 \text { a } 29 \text { años: } 46,2 \% . \\
\text { De } 30 \text { a } 39 \text { años: } 33,6 \% \text {. } \\
\text { De } 40 \text { en adelante: } 12,6 \% .\end{array}$ \\
\hline Sexo & $\begin{array}{l}\text { Mujeres: } 81,8 \% \\
\text { Hombres: } 18,2 \% \text {. }\end{array}$ \\
\hline País de origen & $\begin{array}{l}\text { Polonia: } 13,3 \% \text {. } \\
\text { Bulgaria: } 9,8 \% \text {. } \\
\text { Italia: } 8,4 \% \text {. } \\
\text { China: } 8,4 \% \text {. } \\
\text { El resto de nacionalidades son las siguientes: Alemania y Rumanía (5,6\%), } \\
\text { Francia 4,9\%, Japón, Ucrania, Brasil e Irán (3,5\%), EEUU Grecia y Hun- } \\
\text { gría (2,8\%), Turquía, Argelia e Irlanda (2,1\%), Inglaterra, Eslovaquia, Ma- } \\
\text { rruecos, Rusia y Egipto (1,4\%), Australia, Bélgica, Suiza, India, Holanda, } \\
\text { Libia, Austria, y Túnez (0,7\%). }\end{array}$ \\
\hline Lengua materna & $\begin{array}{l}\text { Las lenguas maternas mayoritarias son el polaco, el búlgaro, el italiano, el } \\
\text { chino y el alemán. }\end{array}$ \\
\hline Nivel de estudios & $\begin{array}{l}\text { Estudios primarios: } 1,4 \% \text {. } \\
\text { Estudios secundarios: } 11,9 \% \text {. } \\
\text { Formación profesional: } 9,8 \% \text {. } \\
\text { Estudios superiores: } 58 \% \text {. } \\
\text { Otros: } 16,8 \% \text {. }\end{array}$ \\
\hline $\begin{array}{l}\text { Estudios previos } \\
\text { de español }\end{array}$ & $\begin{array}{l}\text { SÍ: } 33,3 \% . \\
\text { NO: } 66,7 \% .\end{array}$ \\
\hline
\end{tabular}

1 Agradecemos al Prof. Dr. Mariano del Mazo de Unamuno, director del Departamento de español de la Escuela Oficial de Idiomas «Jesús Maestro» de Madrid y al grupo de profesores y alumnos que han colaborado y facilitado la aplicación de los cuestionarios, la disponibilidad incondicional y el tiempo dedicado.

2 La Escuela Oficial de Idiomas «Jesús Maestro» de Madrid oferta tres modalidades de estudio: presencial, semipresencial y no presencial (a distancia).

3 Para el análisis de los datos, hemos utilizado el programa estadístico SSPS, según las indicaciones del Departamento de Apoyo a la Investigación y a la Docencia de los Servicios Informáticos, Centro de Cálculo de Datos de la Universidad Complutense de Madrid. 


\begin{tabular}{|l|l|}
\hline Otras lengua & $\begin{array}{l}\text { Además de la lengua materna, un } 46,6 \% \text { de los informantes habla inglés, } \\
\text { un } 18,8 \text {, francés; } y \text { un } 29,4 \% \text { dice no hablar otra lengua. El resto del por- } \\
\text { centaje total se completa con otras lenguas en menor proporción: rumano, } \\
\text { ruso, italiano, árabe, irlandés, alemán, polaco. }\end{array}$ \\
\hline $\begin{array}{l}\text { Tiempo viviendo } \\
\text { en España }\end{array}$ & $\begin{array}{l}\text { Menos de } 3 \text { meses: 3,5\%. } \\
\text { De } 3 \text { meses a } 1 \text { año: } 26,1 \% . \\
\text { Más de } 1 \text { año: 70,4\%. }\end{array}$ \\
\hline $\begin{array}{l}\text { Para qué aprenden } \\
\text { español }\end{array}$ & $\begin{array}{l}\text { Gran parte de los encuestados eligió más de una opción, las que presentan } \\
\text { porcentajes más altos son las siguientes: a) para llegar a hablar tan bien } \\
\text { como un español; } b \text { ) para seguir estudiando algo; } c \text { ) para entender a otras } \\
\text { personas d) para encontrar un trabajo; e) para escuchar la radio, entender } \\
\text { la televisión, etc. }\end{array}$ \\
\hline
\end{tabular}

\section{Análisis y discusión de los datos}

En este apartado analizamos y describimos una selección de los datos referidos a las creencias y actitudes de los alumnos ante los errores y la corrección de los mismos en la interacción oral en contexto de aula:

\section{Gráfico 1. Un error es algo grave pero necesario}

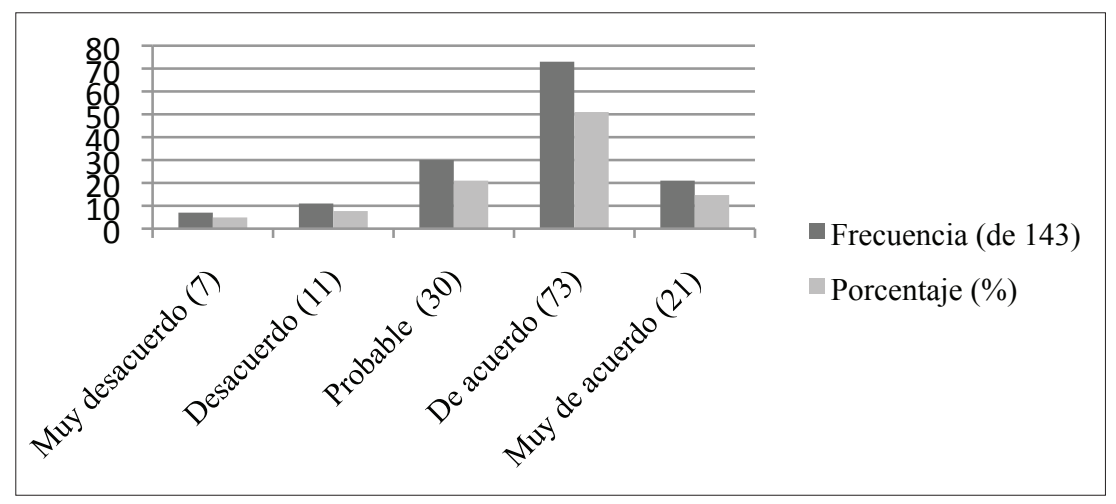

En una muestra tan heterogénea en lenguas de origen y nacionalidades, llama la atención que un $51 \%$ de los participantes exprese estar de acuerdo con una misma teoría implícita. De igual forma, es también reseñable que tan sólo un 4,9\% refleje los enunciados de la lingüística aplicada sobre el error como elemento no grave, pero característico y necesario en el proceso de aprendizaje. 
Gráfico 2. Los errores me impiden aprender

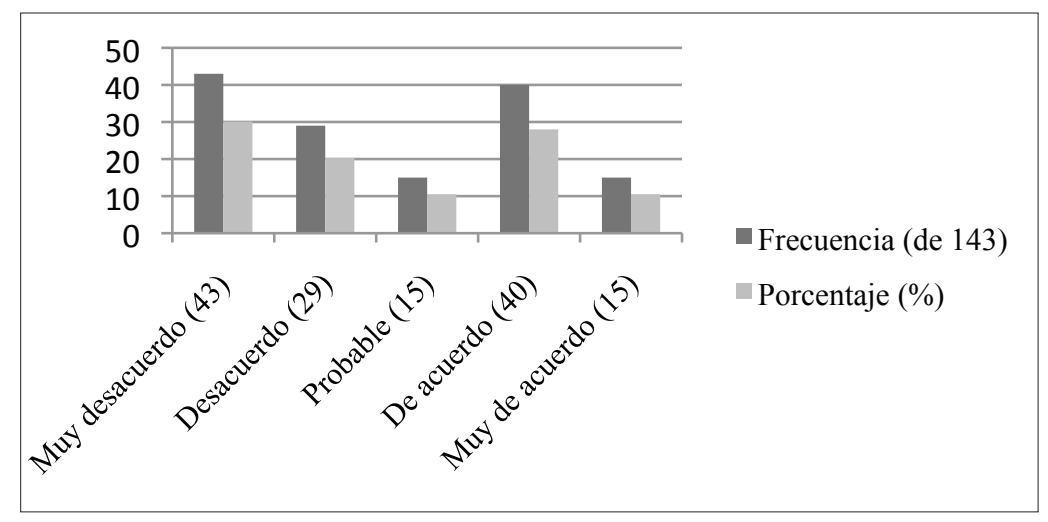

Este ítem muestra una clara división de posturas frente al error como impedimento. El 85\% de la muestra se ubica en esta dicotomía; así, el 50,4\% opina que un error no impide aprender y un 38,5\% se manifiesta de acuerdo o muy de acuerdo en que un error impide aprender más. Cabe reflexionar sobre el porqué de esta distancia respecto a la teoría ampliamente aceptada sobre el error.

Gráfico 3. Un error es algo que no debería ocurrir

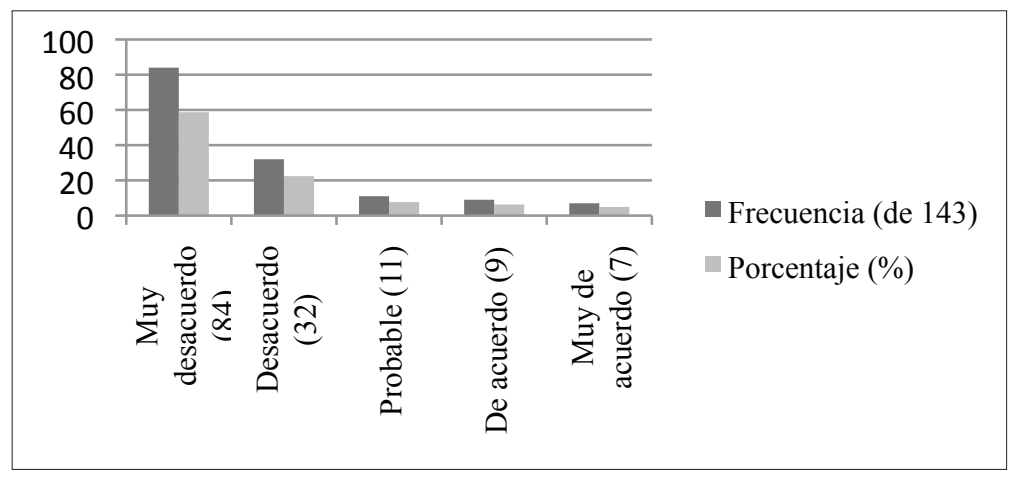

84 muy en desacuerdo y 32 en desacuerdo evidencian un $80 \%$ del total dentro de una misma zona de pensamiento; el consciente colectivo de nuestra muestra percibe el error como fenómeno inevitable y necesario. 


\section{Gráfico 4. Los errores me ayudan a saber cuánto estoy aprendiendo}

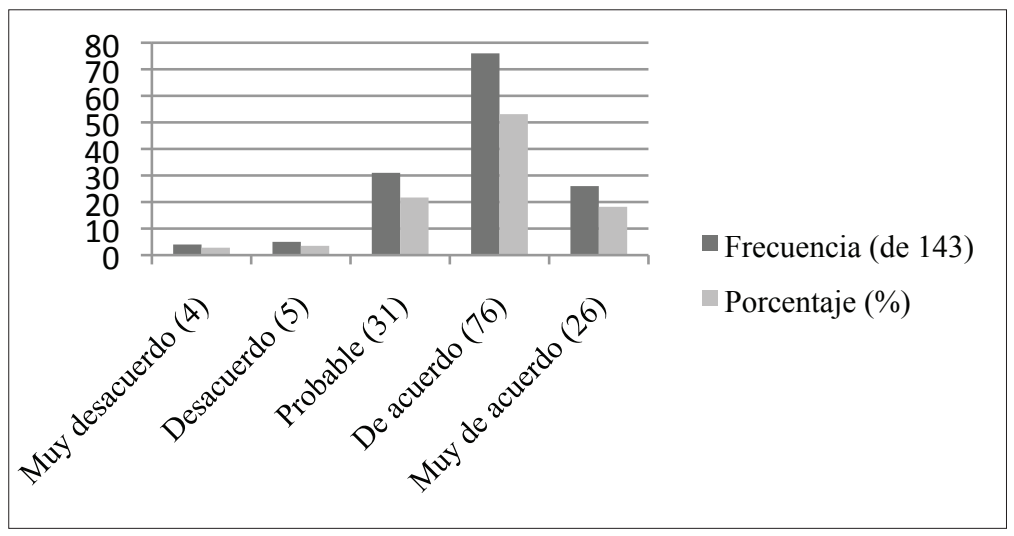

Los errores (comprobación / refutación de hipótesis) denotan que el proceso hacia una L2/LE se está llevando a efecto (Selinker, 1972); aunque un 6,3\% no lo considere así, un $71,3 \%$ sí que considera el error como elemento indicador de su proceso de aprendizaje hacia la lengua meta

\section{Gráfico 5. Uso mi español incluso si cometo errores}

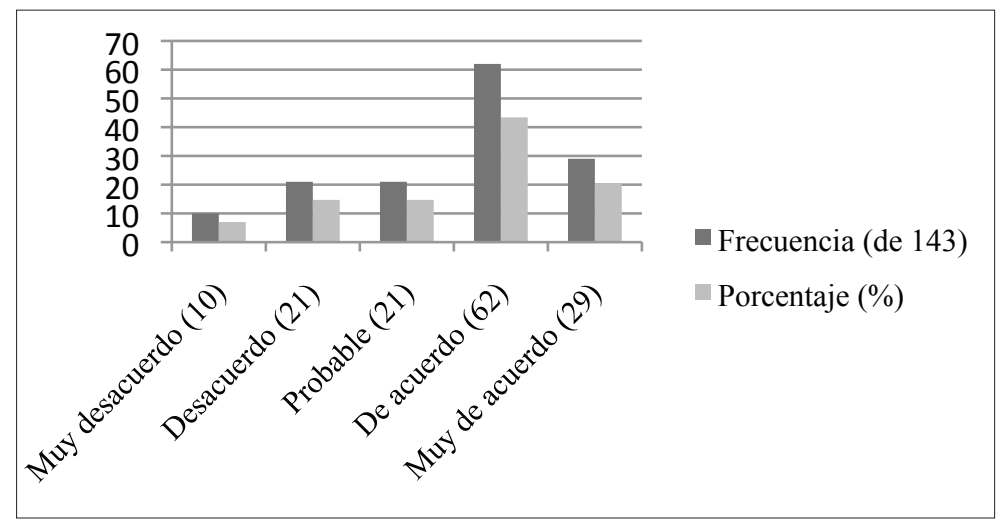

Un $80 \%$ de los participantes en el estudio admite el error dentro de lo aceptable y probable. En este ítem, el $63 \%$ de la muestra usa su español incluso si comete errores. En esta línea, Fernández de Amo (2008) desarrolla el concepto de error afectivo, para referirse a aquellos errores que no responden a la falta de conocimiento o competencia sino a timidez, inhibición o miedo a exponerse. 
Gráfico 6. Me gusta que me corrijan

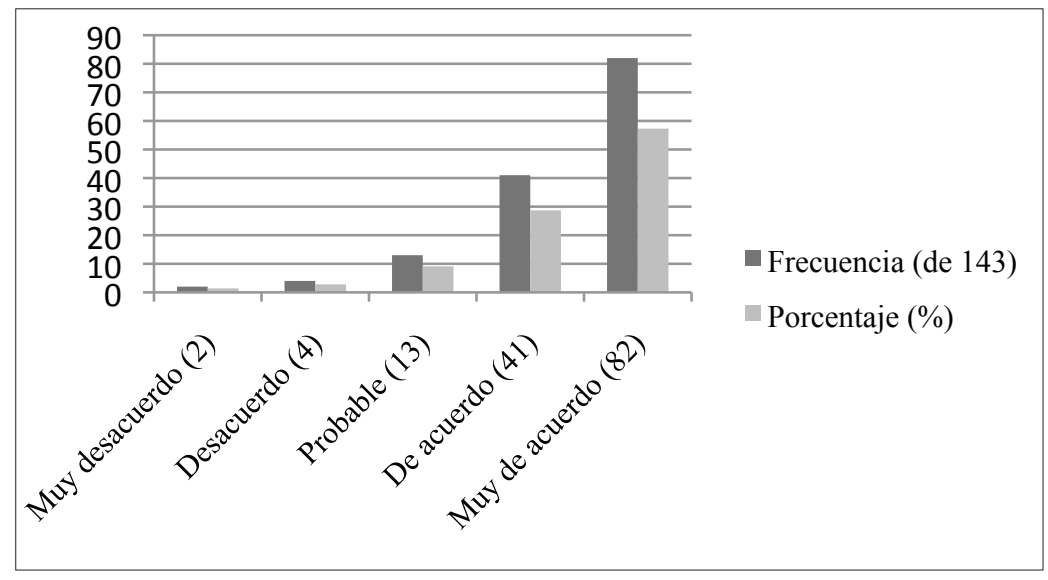

Un $86 \%$ de los informantes admite que les gusta ser corregidos. Es uno de los porcentajes más altos dentro del todas las variables, por lo que podemos considerar este dato como tendencia significativa.

Gráfico 7. Me gusta que me corrija el profesor

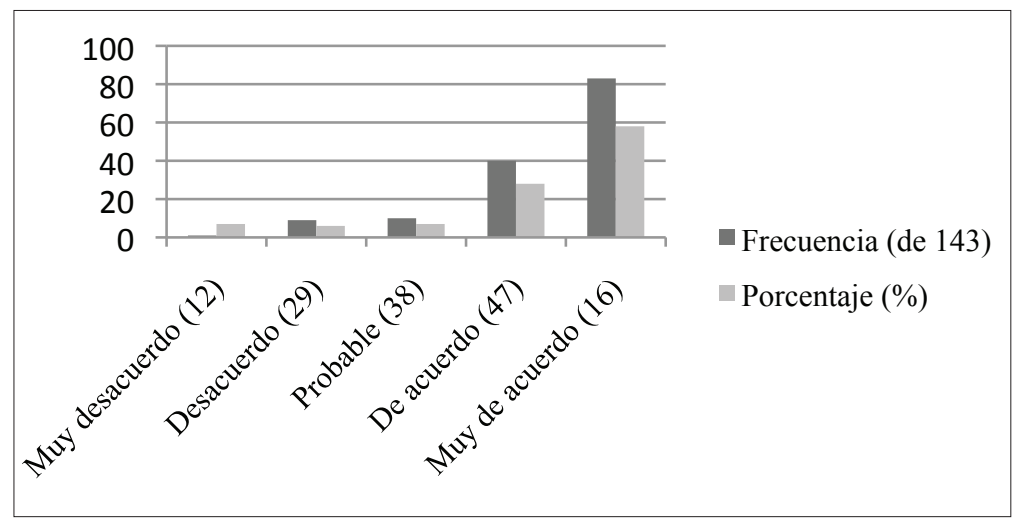

Los informantes, agrupados en un $86 \%$ (muy de acuerdo: $58 \%+$ de acuerdo: $28 \%$ ) nos revelan cómo la tradición del profesor como vehículo de la corrección en clase está presente. Las representaciones o creencias permean nuestras maneras de reaccionar y actuar en situaciones de la vida cotidiana y no deja indiferente el $14 \%$ de la muestra que ha respondido a muy en desacuerdo, desacuerdo o probable. 
Gráfico 8. Me gusta que me corrijan mis compañeros

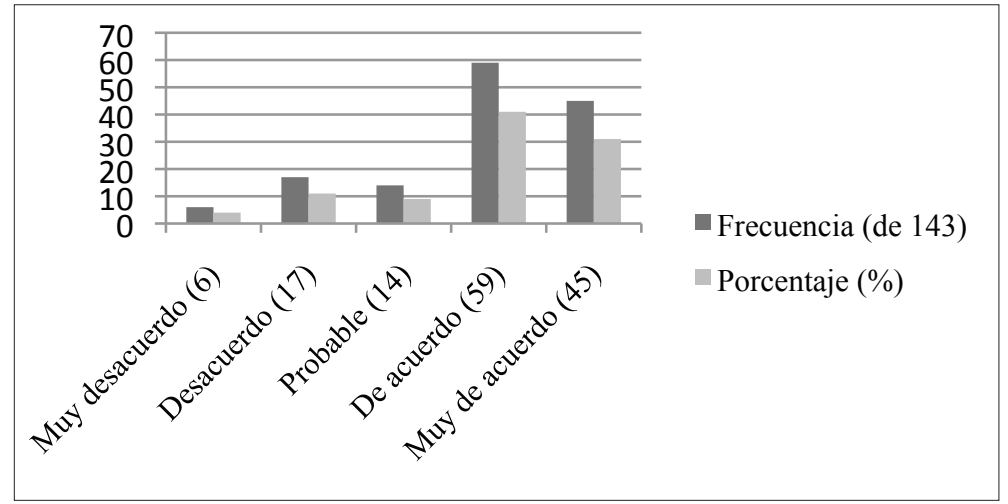

Una actitud positiva ante el aprendizaje de lenguas está relacionada con la apertura hacia nuevas experiencias, hacia otras personas (MCER, 2002). Por tanto, subrayamos el $16,1 \%$ de informantes (muy desacuerdo: 4,2\%; desacuerdo $11,9 \%)$ que coinciden en la zona de la muestra reticente a permitir que un compañero de clase actúe como agente de corrección. La tendencia general (72,8\%), sin embargo, se concentra en la zona y actitud opuestas.

Gráfico 9. Me gusta que me corrijan el vocabulario

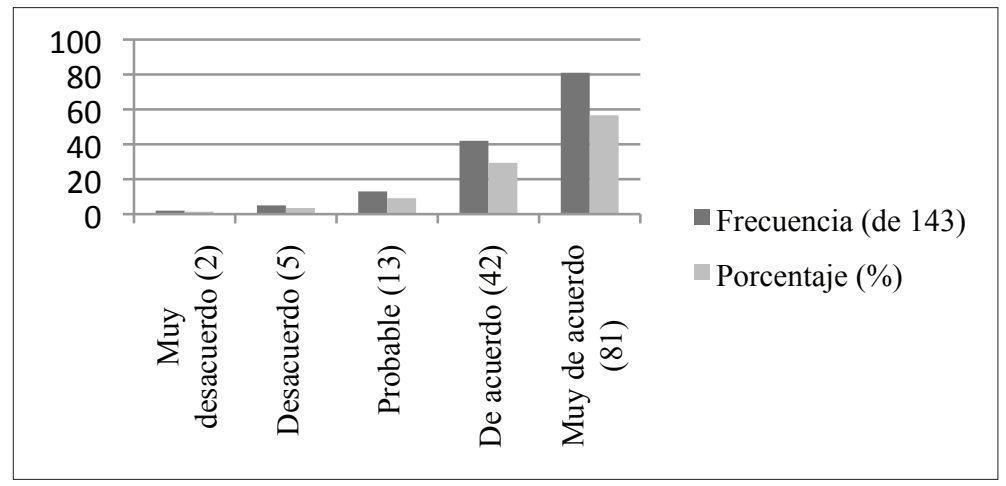

La zona muy de acuerdo $(56,6 \%)+$ de acuerdo $(29,4 \%)$ concentra al 86\% de la población de muestra. 
Gráfico 10. Me gusta que me corrijan la pronunciación

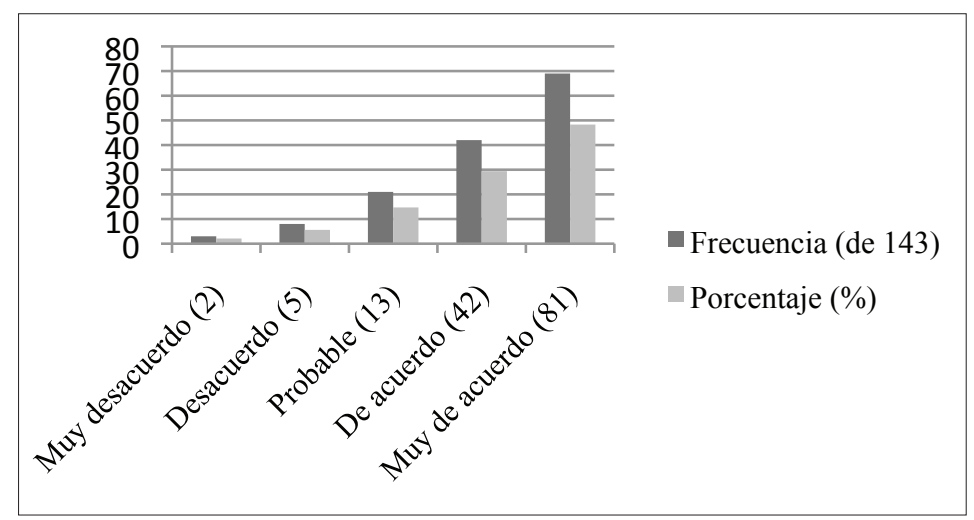

Para Díaz Martínez (1998) la falta de afectividad y empatía por parte del profesor ante la corrección de los errores en la producción oral - especialmente en la pronunciación - produce en el aprendiente falta de receptividad, entre otros. Nuestra muestra está compuesta, sin embargo por un $77,7 \%$ que se manifiesta de acuerdo en que la pronunciación sea corregida, frente a un $7,7 \%$ que claramente no lo desea tan abiertamente y un $14,7 \%$ que lo considera probable.

Gráfico 11. Me gusta que me corrijan la gramática

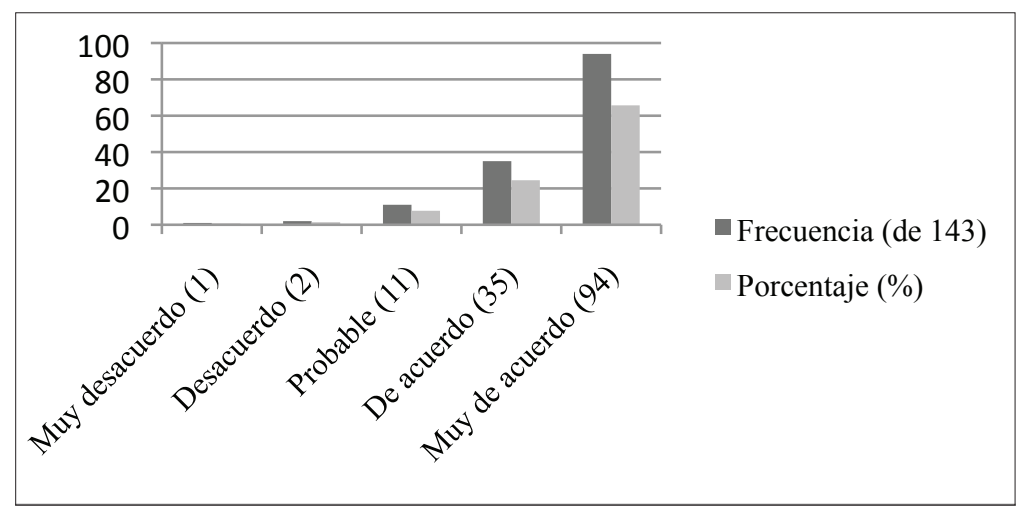

En este ítem encontramos el más alto porcentaje de muy de acuerdo, en total, el $65,7 \%$. No resulta infrecuente que tanto profesores como alumnos den prioridad y consideren más graves los errores que atañen a la lengua como sistema gramatical. 
Gráfico 12. Me gusta que me corrijan la adecuación

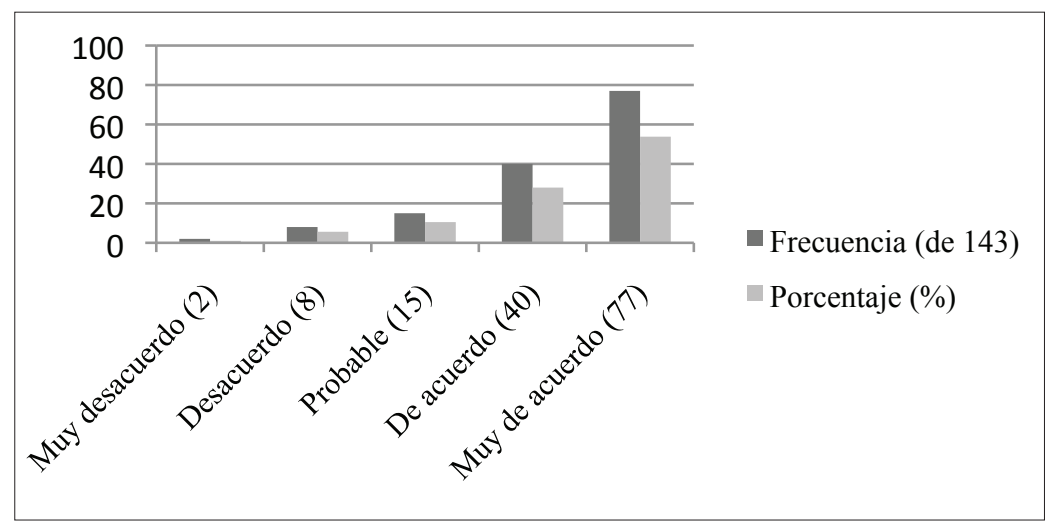

Consideramos los errores como desviaciones de la norma en aspectos también concernientes a la adecuación al contexto y a las normas socioculturales, aspecto en el que destaca un $81,8 \%$ que dice estar muy de acuerdo $(53,8 \%)$ y de acuerdo (28\%) en ser corregido.

Gráfico 13. Me gusta que me corrijan cuando he terminado de hablar

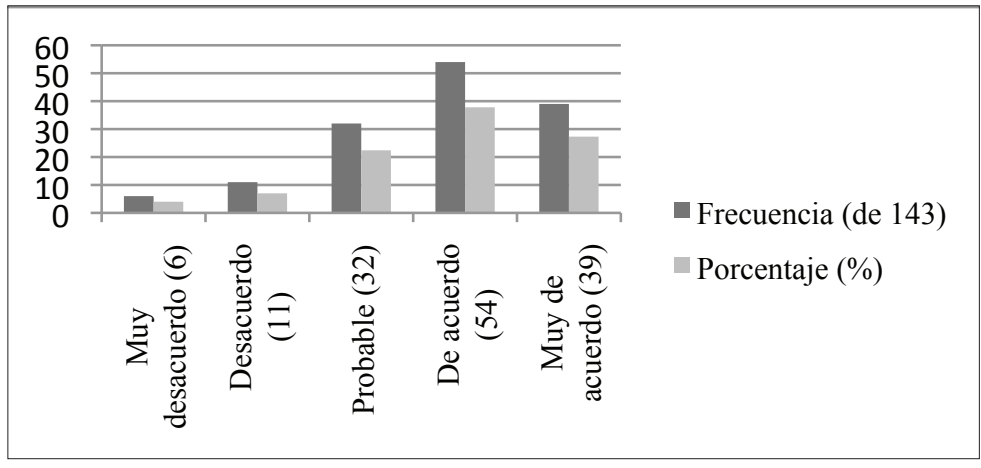

Aunque muy de acuerdo (27,3\%) y de acuerdo (37,8\%) presentan porcentajes interesantes, pues apoyan la técnica de corregir después de la producción, es igualmente reseñable el $22,4 \%$ casi una cuarta parte, que manifiesta probable la aceptación de este tipo de técnica correctiva. 
Gráfico 14. Me gusta que me corrijan mientras estoy hablando

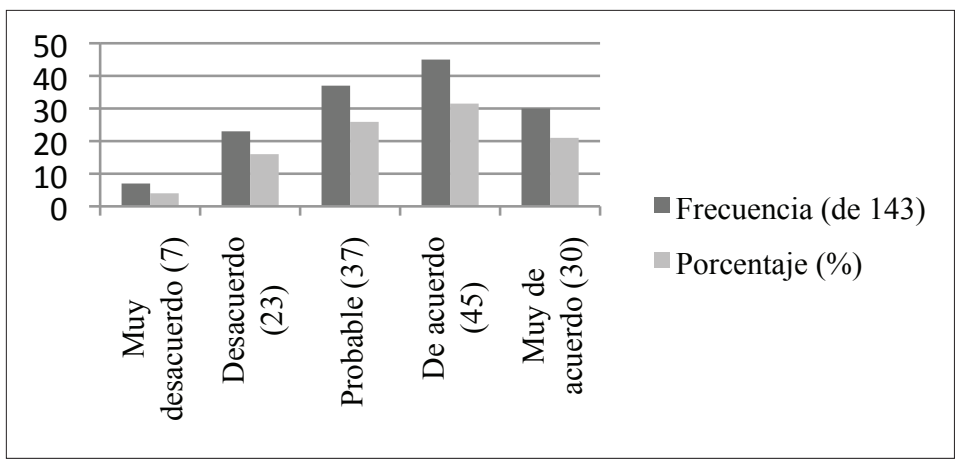

Barbero Carcedo (2012) sistematiza y agrupa las técnicas correctivas orales en dos variables principales - a) interrumpir al alumno o no y $b$ ) dar la solución o no. A la corrección mientras se está hablando, observamos aquí que un 25,9\% responde probable. Igualmente, más de la mitad de los informantes 52,5\% (muy de acuerdo $21 \%$ y de acuerdo $31,5 \%$ ) prefieren ser corregidos mientras están hablando en clase, cifras que nos revelan una tendencia a la metodología tradicional.

\section{Gráfico 15. Me gusta que me corrijan cada vez que cometo un error}

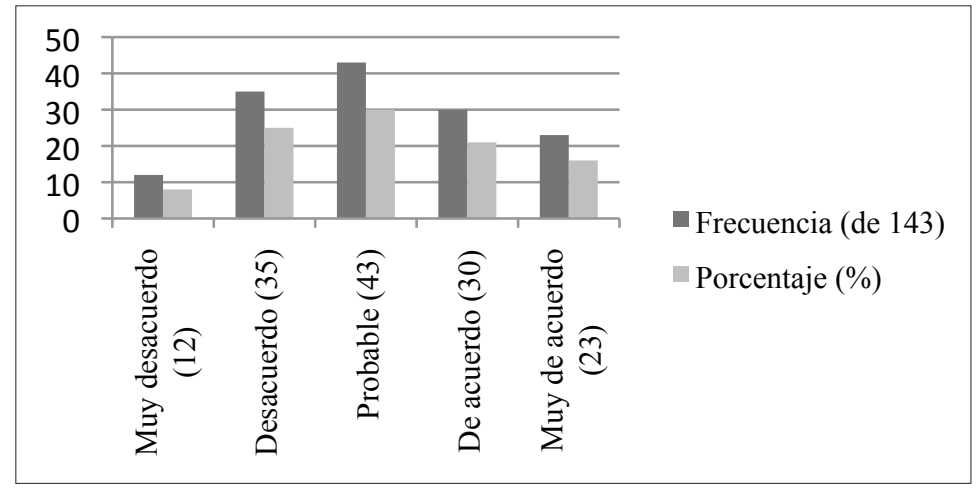

En este ítem encontramos el porcentaje más alto de todas las manifestaciones de desacuerdo (24,5\%) en el conjunto de la encuesta. Constituye casi una cuarta parte de la población encuestada, lo que nos hace pensar que existe un importante consenso en la actitud frente al cuándo corregir. Aun así, un 30\% ha respondido probable y un nada desdeñable $37,1 \%$ se ubica en la zona de muy de acuerdo $(16,1 \%)$ o de acuerdo (21\%), datos que nos muestran que el ser corregido con intensidad y recurrencia prevalece entre las preferencias de nuestros alumnos. 


\section{Gráfico 16. Me gusta que me den la palabra correcta}

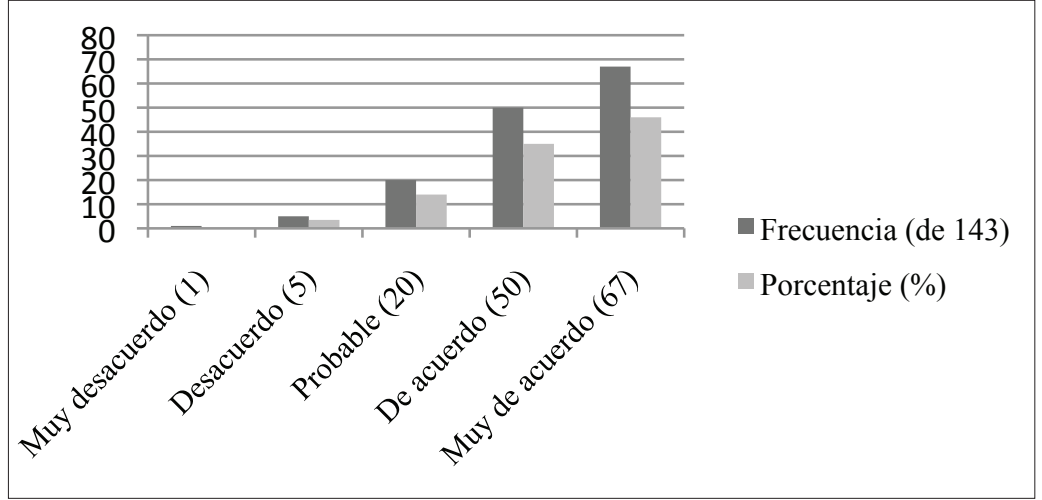

El gráfico deja constancia de que un altísimo porcentaje de los alumnos manifiesta su preferencia por la corrección completa frente a las indicaciones para llevar a cabo la autocorrección.

Gráfico 17. Me gusta reparar yo mismo mis errores

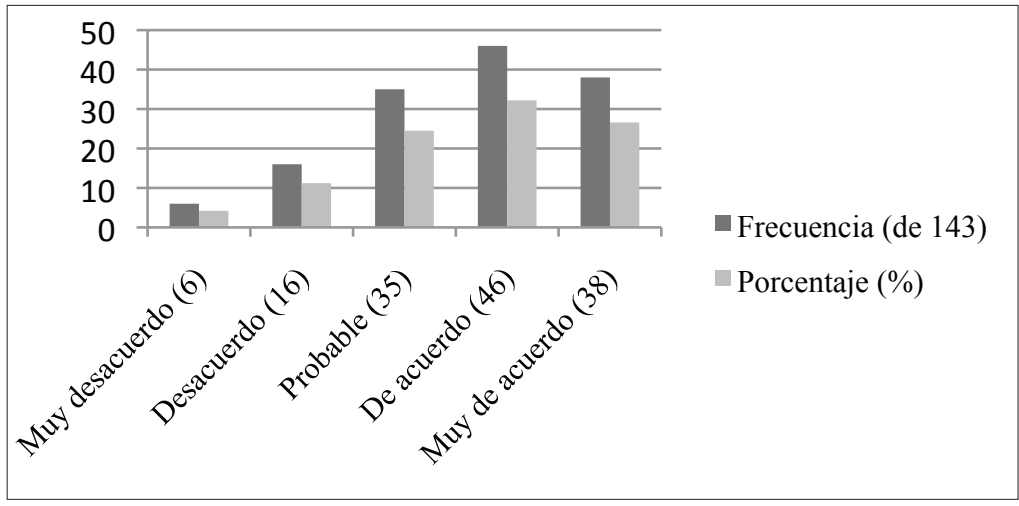

A pesar de lo constatado en las respuestas al ítem anterior, un 58,8\% de la muestra también se manifiesta partidario de la autocorrección. 
Gráfico 18. Me gusta que me graben en clase para detectar mis propios errores

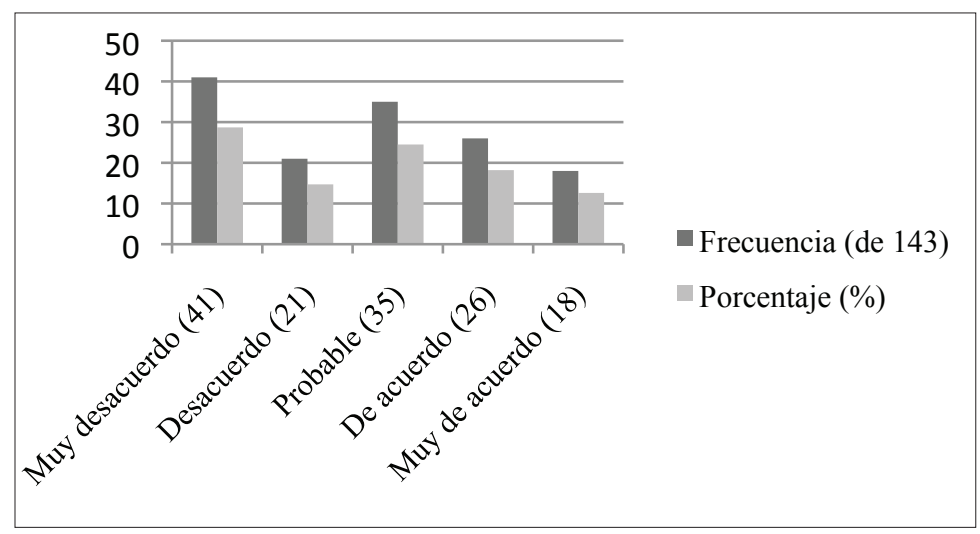

El MCER (2002) considera que una actitud positiva ante el aprendizaje de lenguas está relacionada con la apertura hacia nuevas experiencias. Las técnicas correctivas que explora este ítem no son aceptadas por un $42,4 \%$, con un $28,7 \%$ que está muy en desacuerdo.

\section{Conclusiones}

Los datos analizados nos permiten responder, si bien con cierta cautela, a los objetivos planteados al inicio de nuestro estudio. En primer lugar, en relación con la actitud positiva/negativa de los alumnos ante los errores, parece darse una postura en cierto modo neutra, pues si bien los consideran necesarios para aprender más y mejor, también creen que son algo grave (73\%); esta opinión, en cierto modo contradictoria, se ve avalada por los resultados de las otras variables referidas a este mismo aspecto.

Referente a la actitud ante la corrección de los errores y en consonancia con la creencia de nuestros informantes de que son algo grave en su interlengua y que no deberían ocurrir en un estudiante, un alto porcentaje de los estudiantes, casi la totalidad $(82 \%$, ) gusta abiertamente de ser corregido, de lo que se deduce que la creencia sobre el concepto de error actúa sobre su actitud ante la corrección de los mismos en la interacción oral.

En consonancia con lo anterior y en relación con quién debe corregir manifiestan su acuerdo en que sea el profesor tanto como sus compañeros, en cualquier caso, que la corrección se efectúe. Asimismo, la tradición se manifiesta también en el qué corregir, ya que los porcentajes relativos a la gramática, el vocabulario y 
la pronunciación sobrepasan la media, aunque no se ignora tampoco, la adecuación pragmática y discursiva: quieren ser corregidos.

En lo relativo a cuándo ser corregidos, un interesante $67,1 \%$ declara que le gusta que le corrijan mientras está hablando y un 55\% de la misma muestra admite que le gusta ser corregido después de haber terminado de hablar; de lo que se deriva que un número importante de alumnos no tiene inconveniente en ser interrumpido.

Finalmente, en relación con las distintas técnicas empleadas por el profesor, no existe una preferencia destacada por ninguna de las variables incluidas en nuestro estudio, sino que las preferencias se distribuyen entre todas ellas excepto en el caso de «detener cada vez que se comete un error y dar la forma correcta» (46\%).

A tenor de los resultados específicos en lo concerniente a la relación entre las teorías implícitas y las actitudes de los alumnos, podemos colegir que las creencias - vinculadas a experiencias previas, a su cultura educativa y quizá a la actuación del profesor- inciden en una actitud tradicional ante los errores y la corrección de los mismos, lo cual puede estar en consonancia también con los principales motivos por los que aprenden español, comentados en el apartado dedicado al perfil de la muestra y entre los que destacamos: a) para llegar a hablar tan bien como un español; $b$ ) para entender a otras personas; $c$ ) para encontrar un trabajo; d) para escuchar la radio, entender la televisión, etc., propósitos para los que consideran importante la corrección idiomática.

No ignoramos que la actitud del profesor condiciona la del alumno en gran medida, por lo que sería necesario incluir datos a este respecto y correlacionarlos con los del alumno, aspecto que desarrollaremos en nuestro próximo estudio.

\section{Referencias bibliográficas}

Abelson, R. (1979). «Differences between beliefs and knowledge systems». Cognitive Science, 3 (4): 355-366.

Arumi Ribas, M. (2009). «Estudio de las percepciones de los estudiantes de lenguas extranjeras sobre el uso de instrumentos de autorregulación». RES$L A, 22: 35-58$.

Barbero Carcedo, V. (2012). «¿Cómo acertar en la corrección? Ventajas e inconvenientes de las técnicas de corrección en la interacción oral». Boletín de la Asociación para la Enseñanza del Español como Lengua Extranjera, 46: 17-30.

Chaparro, M. (2008). Estudio descriptivo de la actitud del hablante no nativo de español ante la corrección de errores en la interacción oral. Trabajo de Investigación inédito. Madrid: Universidad Complutense. 
Chaudron, C. (1988). Second Language Classroom. Research on Teaching and Learning. Cambridge: Cambridge University Press.

Consejo de Europa (2002). Marco común europeo de referencia para las lenguas: aprendizaje, enseñanza, evaluación. Madrid: Ministerio de Educación, Cultura y Deporte - Instituto Cervantes - Editorial Anaya [MCER].

Corder, S.P. (1967). «The Significance of learner's errors»+ International Review of Applied Linguistics, 5: 161-170. (Traducción al español en J. Muñoz LICERAs (Ed.) (1991). La Adquisición de las lenguas Extranjeras. Madrid: Visor: 31-40).

- (1981). Error Analysis and Interlanguage. Oxford: Oxford University Press.

Díaz Martínez, J. (1998). Afectividad y tratamiento del error en la clase de segundas lenguas: estudio introspectivo de un diario. Barcelona: Universidad de Barcelona.

Fernández de Amo, M. (2008)+ «Una visión afectiva del error oral en el aula de inglés», Revista Digital «Práctica Docente», 9: 39-48.

Fernández López, S. (2000). «Corrección de errores en la expresión oral». Carabela, 47: 133-150.

Groult, N. (2005). «Para mí, aprender una lengua significa... Representaciones de alumnos universitarios mexicanos acerca de las lenguas extranjeras». ELIA6: 93-114.

Horwitz, E. K. (1987). «Surveying students' beliefs about language learning» en A. Wenden y J. Rubin (Eds). Learner's Strategies in Language Learning. Englewood Cliffs, NJ: Prentice-Hall: 119-129.

Larsen Freeman, D. y Long, M. H. (1994). Introducción al estudio de la adquisición de segundas lenguas. Madrid: Gredos.

Long, M. H. (1977). «Teacher feedback on learner errorः mapping cognitions». TESOL, 77: 278-294.

Martín Peris, E. (Dir.) (2008). Diccionario de términos clave de español como lengua extranjera. Madrid: SGEL [DTCELE].

Martínez Abellán, A. (1999). «Componente actitudinal en la enseñanza de idiomas». Comunidad Educativa, 258:22-25.

Mekassimove, S. (2009). «Las creencias de los alumnos de la secundaria gabonesa acerca de su proceso de aprendizaje del español/LE». MarcoELE, 9: $1-17$.

Nunan, D. (1992). Research Methods in Language Learning. Cambridge: Cambridge University Press.

Pajares, M. F. (1992). «Teacher's beliefs and educational researchः cleaning up a messy construct». Review of Educational Research, 62 (3): 307-332. 
Picó, E.; Verdés, G. y Vilagrasa, A. (1990). «Las teorías implícitas, estrategias y preferencias de los estudiantes de E/SL en relación a su nivel de toma de riesgo. Algunas correlaciones e implicaciones pedagógicas». Actas del I Congreso Nacional de Asele. Granada: 241-253.

Richards, J.C. y Lockhart, C. (1998). Estrategias de reflexión sobre la enseñan$z a$ de idiomas. Cambridge: Cambridge University Press.

Roncel Vega, V. M. (2008). «Autoconcepto, motivación y ansiedad en el aula de idiomas», MarcoELE, 7: 1-19.

Santos Gargallo, I. (1993). Análisis contrastivo, análisis de errores e interlengua en el marco de la lingüística contrastiva. Madrid: Síntesis.

- (2004). «Análisis de errores en la interlengua de hablantes no nativos (HNN) de español» en J. Sánchez Lobato e I. Santos Gargallo (Eds.), Enseñar español como segunda lengua/lengua extranjera. Vademécum para la formación de profesores. Madrid: SGEL: 391-410.

Selinker, L. (1972). «Interlanguage». International Review of Applied Linguistics, 10:209-231. (Traducción al español: «La interlengua» en J. Muñoz Liceras (1991) (Ed.), La adquisición de lenguas extranjeras. Madrid: Visor: 79-101).

Serra, R. (1997). «Las representaciones de los profesores sobre la enseñanzaaprendizaje de lenguas. Estado de la cuestión desde la perspectiva institucional del currículum de lenguas», en M. L. Villanueva e I. Navarro (Eds.), Los estilos de aprendizaje de lenguas. Castelló: Collección Summa: 155-175. YIN, R. (1984). Case Study Research: Design and Methods. Beverly Hills: SAGE. 